\title{
Infrared spectra of silica polymorphs
}

\author{
Chiyoe Koike ${ }^{1}$ Ritsumeikan University, Kusatsu, Shiga 525-8577, Japan \\ E-mail: koike-c@mua.biglobe.ne.jp \\ Ryo Noguchi Osaka University, Toyonaka, Osaka 560-0043, Japan \\ Hiroki Chihara Osaka Sangyo University, Daito, Osaka 574-8530, Japan \\ Hiroshi Suto National Astronomical Observatory of Japan, Mitaka, Tokyo 181-8588, Japan \\ Osamu Ohtaka, Yuta Imai,Tooru Matsumoto \\ Osaka University, Toyonaka, Osaka 560-0043, Japan \\ Akira Tsuchiyama Kyoto University, Kyoto 606-8052, Japan
}

The existence of silica within several debris disks has been suggested. We investigate the annealing conditions of $\alpha$-cristobalite, and further prepare various types of silica, including $\alpha$-cristobalite, $\alpha$-quartz, coesite, stishovite, and fused quartz, which are natural, synthetic or commercial samples. We compare the results to previous studies and find that $\alpha$-cristobalite synthesized at higher temperature than annealed silica. The interesting result of features similar to those of forsterite should be highlighted, where $\alpha$ cristobalite and coesite showed similar peaks at 16,33 , and $69 \mu \mathrm{m}$ as forsterite. The $69 \mu \mathrm{m}$ band for $\alpha-$ cristobalite is especially very broad and strong, and shifts largely to a shorter wavelengths under cooling to low temperatures. The band for coesite, however, is very sharp, and shifts only a small amount to longer wavelengths under cooling to low temperatures. The peak positions of 16 and $69-\mu \mathrm{m}$ band due to $\alpha$-cristobalite can become index for temperature of silica dust. We discuss the possibility of silica detection around debris disks.

The Life Cycle of Dust in the Universe: Observations, Theory, and Laboratory Experiments (LCDU 2013 ) November 18-22, 2013

Taipei, Taiwan

$1 \quad$ Speaker 


\section{Introduction}

Recently, certain unusual features were detected in debris disks HD 172555, HD 15407A, HD 23514A and HD 23514, in which prominent peaks appeared at about 9.3, 12, and $21 \mu \mathrm{m}$ [1] and at about 9, 16 and 20-22 $\mu \mathrm{m}[2]$. The unusual spectra of HD 15407 observed by Fujiwara et al. (2012)[3] were particularly notable, as two significant emission peaks occurred at about 9 and $20 \mu \mathrm{m}$, and a weak peak at $16 \mu \mathrm{m}$ was also detected. The spectra fit well with amorphous silicate (amorphous pyroxene), fused silica and annealed silica. The annealed silica explains the feature observed at $16 \mu \mathrm{m}$, while producing a sharp extra emission at $12.6 \mu \mathrm{m}$ [3]. Similar results reported a significant fraction of $\mathrm{SiO}_{2}$ (annealed silica as cristobalite) is required, fitting the observed spectrum of HD 15407[4]. However, the laboratory data that exists in the literature neither covers broad enough a spectral range, nor enough silica polytypes to achieve this goal. In this study, we measured the infrared spectra of the various silica polymorphs over a broad range of wavelengths, i.e., in mid- and far-wavelength regions. Following a description of our results for the various silica polymorphs compared with previous studies, we discuss the possibility of silica detection around debris disks.

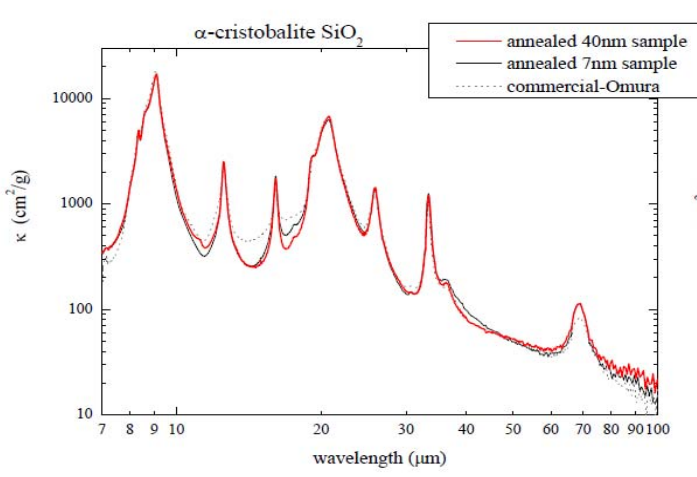

(a)

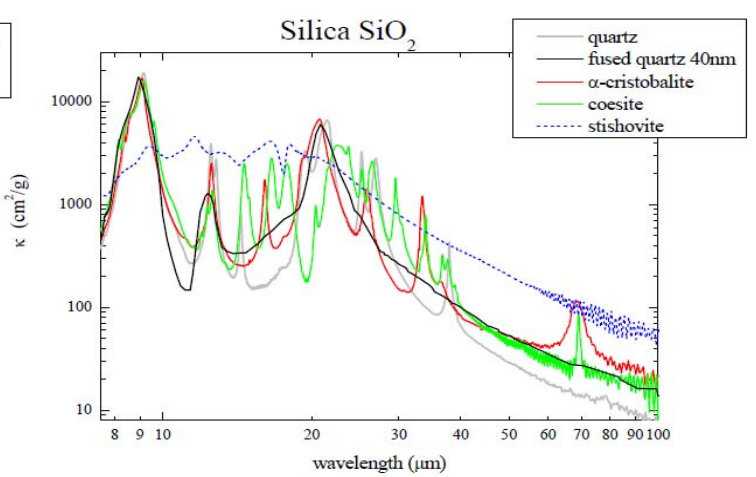

(b)

Fig. 1. The spectra of $\alpha$-cristobalite samples (a) and all samples (b).

\section{Sample preparation and results}

We prepared many types of silica polymorphs including $\alpha$-cristobalite, fused quartz, $\alpha$ quartz, coesite, and stishovite, obtained both in the laboratory and from commercial and natural samples. Particularly, the $\alpha$-cristobalite sample was prepared by annealing commercial fused quartz; fine powder of fused quartz was annealed at $1773 \mathrm{~K}$ for 3 and 6 days and confirmed through X-ray diffraction (XRD), which is higher than $1220 \mathrm{~K}$ for the annealed silica[5]. A commercial sample of finely powdered $\alpha$-cristobalite was obtained from Omura Ceratec Inc., Japan. The fine particles of the silica samples were well dispersed in $\mathrm{KBr}$ and polyethylene (PE) powder and were pressed, respectively, into $\mathrm{KBr}$ pellets and $\mathrm{PE}$ sheets, whose transmittance was measured with a Nicolet 670 and/or 6700 in the mid- and far-infrared regions. The mass absorption coefficient $\kappa$ was derived from the transmittance T. The spectra of $\alpha$-cristobalite and all samples are shown in Fig.1(a) and (b), respectively. The strength and peak position of the 9$\mu \mathrm{m}$ band were very similar for all samples except stishovite. However, many sharp peaks 
appeared in different bands for coesite and quartz. In the far-infrared region at approximately 69 $\mu \mathrm{m}$, a strong and broad band appeared for $\alpha$-cristobalite, and a strong and sharp peak appeared for coesite.

\section{Spectra at low temperatures compared with those of forsterite}

The spectra of $\alpha$-cristobalite and coesite showed similar bands at 16,33 and $69 \mu \mathrm{m}$ to those of forsterite, as shown in Fig. 1. The mineral forsterite is a significant component in circumstellar dust and is ascertained by the peak positions of the observed infrared spectra.

Magnesium silicate $\left(\mathrm{MgSiO}_{3}\right)$ smokes, after annealing, were transformed to crystalline forsterite and tridymite or cristobalie [5], [6]. If amorphous magnesium silicate grains were heated by central star, it may be possible that forsterite and $\alpha$-cristobalite grains coexist in the circumstellar environment. To discriminate between the samples ( $\alpha$-cristobalite and coesite) and forsterite, the spectra of coesite and $\alpha$-cristobalite were measured at low temperatures from room temperature (RT) to $8 \mathrm{~K}$, and the peak positions of $\alpha$-cristobalite and coesite were compared with those of forsterite. Most peak positions of each band shifted to shorter wavelengths and became sharper as the samples cooled from RT to $8 \mathrm{~K}$; however, several peak positions shifted to longer wavelengths. In the case of $\alpha$-cristobalite, the peak positions of the 33 and $36 \mu \mathrm{m}$ bands shifted to longer wavelengths as the temperature decreased. In the case of coesite, the peak positions of the 34, 38, 39 and $69 \mu \mathrm{m}$ bands shifted to longer wavelengths with decreasing temperature. The differences among $\alpha$-cristobalite, coesite and forsterite are clear for the $16 \mu \mathrm{m}$ band, as indicated by their distinguished peak positions. For the $33 \mu \mathrm{m}$ band, the peaks of $\alpha$-cristobalite and coesite were sharper than that of forsterite. For the $69 \mu \mathrm{m}$ band as shown in Fig. 2, the band of $\alpha$-cristobalite was very broad, and its peak position differed significantly from that of forsterite, that is, peak positions shifted largely to shorter wavelength as temperature decreased. Although the peak position was the same at $200 \mathrm{~K}$, the band for coesite was sharper and shifted to a longer wavelength than that of forsterite at lower temperature[7].

\section{Detection of silica in debris disks}

Around T Tauri stars (TTSs), annealed silica (cristobalite and/or tridymite) is apparent from mid-infrared observations, in which annealing of amorphous silica near TTSs was first proposed[6]. In this case, the important point is to determine the annealing temperature whether the temperature of the circumstellar disk can be raised to above 1673-1773 K instead of 1220 $\mathrm{K}$ [5] (for annealed silica) by the heating up of the central star; otherwise, an additional process such as heating by collision must be introduced to explain the existing peak of the $16 \mu \mathrm{m}$ band of $\alpha$-cristobalite. Annealing amorphous magnesium silicates changed to forsterite, tridymite, $\alpha$-cristobalite and enstatite[5]. From these results, forsterite and $\alpha$-cristobalite may exist together around disks. For the evidence of existing $\alpha$-cristobalite, the broad and strong band at approximately 69-62 $\mu \mathrm{m}$ could be detected through more detailed observations in far-infrared regions. If mineral dust of coesite and $\alpha$-cristobalite could exist near debris disks after being produced by high-speed collisions among planetesimals, then this characteristic band could be 
detected by high-resolution observations in the $60-70 \mu \mathrm{m}$ regions, although the presence of cold dust has not been confirmed around the outer region disk of the HD 15407.

The $69-62 \mu \mathrm{m}$ band is highly sensitive to the ambient temperature; that is, this peak clearly appeared below approximately $540 \mathrm{~K}$ (phase transition temperature)[8]. Detection of the 16- $\mu \mathrm{m}$ peak in the debris disks also depends on the ambient temperature in the circumstellar disk, where $\alpha$-cristobalite clearly shows a peak at $16 \mu \mathrm{m}$ at temperature below $\sim 540 \mathrm{~K}$. Thus, detection of the 16- $\mu \mathrm{m}$ peak reveals that the ambient temperature may be under $540 \mathrm{~K}$. If the temperature exceeds the transition temperature of approximately $500-550 \mathrm{~K}, \alpha$-cristobalite alters to $\beta$-cristobalite in the phase diagram, and the peaks at 16, 26, 33 and $69 \mu \mathrm{m}$ disappear[8], [9]. The 26, 33 and $69-\mu \mathrm{m}$ peaks may appear or disappear with the grain temperature like the $16-\mu \mathrm{m}$ peak. The $16-\mu \mathrm{m}$ peak disappears after heating above $\sim 540 \mathrm{~K}$ and then appears after cooling below $\sim 540 \mathrm{~K}$. The transition is completely reversible and thermal hysteresis displays[9]. The observations of these peaks of 16,33 , and $69-\mu \mathrm{m}$ band (peak position and FWHM) may become a diagnostic for characteristic grain temperatures like the 69$\mu \mathrm{m}$ band of forsterite.

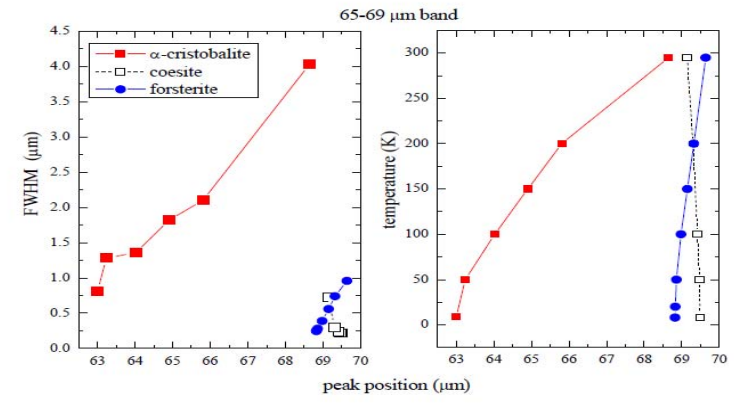

(a)

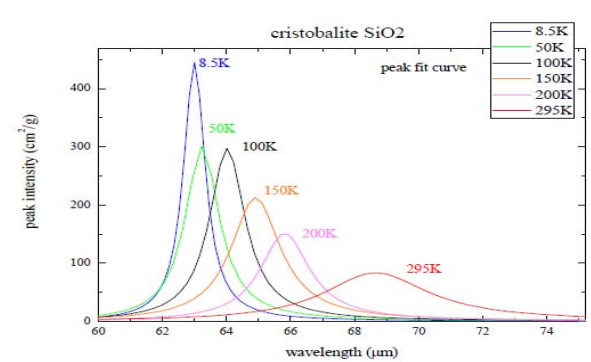

(b)

Fig. 2. The $69-\mu \mathrm{m}$ band of $\alpha$-cristobalite. (a) Peak position dependence on the FWHM of band (left) and on temperature (right) compared with those of coesite and forsterite. (b) The 69$\mu \mathrm{m}$ band of $\alpha$-cristobalite showing a shift to short wavelengths as temperature decreases.

\section{References}

[1] Lisse C.M., Chen C.H., Wyat M.C., et al., 2009, APJ, 701, 2019

[2] Melis C., Zuckerman B., Rhee J., and Song I., 2010, , APJ 717, L57

[3] Fujiwara H., Onaka T., Yamashita T., et al., 2012, APJ, 749, L29

[4] Olofsson J., Juhász A., Th. Henning, et al., 2012 , A\&A 542, 90

[5] Fabian D., Jäger C., Henning T., et al., 2000, A\&A, 364, 282

[6] Sargent B., Forrest W., Tayrien C., et al., 2009, APJ 690, 1193

[7] Koike C, Noguchi R., Chihara H., et al., 2013, APJ, 778:60

[8] Zhang M., \& Scott J.F., 2007, J. Phys.: Condens. Matter 19, 275201

[9] Finnie K.S., Thompson J.G. \& Withers R.L., 1994, J. Phys. Chem. Solids 55, 23 